\title{
Corticostriatal Divergent Function in Determining the Temporal and Spatial Properties of Motor Tics
}

\author{
-Michal Israelashvili and @Izhar Bar-Gad \\ The Leslie and Susan Goldschmied (Gonda) Multidisciplinary Brain Research Center, Bar-Ilan University, Ramat-Gan 52900, Israel
}

Striatal disinhibition leads to the formation of motor tics resembling those expressed during Tourette syndrome and other tic disorders. The spatial properties of these tics are dependent on the location of the focal disinhibition within the striatum; however, the factors affecting the temporal properties of tic expression are still unknown. Here, we used microstimulation within the motor cortex of freely behaving rats before and after striatal disinhibition to explore the factors underlying the timing of individual tics. Cortical activation determined the timing of individual tics via an accumulation process of inputs that was dependent on the frequency and amplitude of the inputs. The resulting tics and their neuronal representation within the striatum were highly stereotypic and independent of the cortical activity properties. The generation of tics was limited by absolute and relative tic refractory periods that were derived from an internal striatal state. Thus, the precise time of the tic expression depends on the interaction between the summation of incoming excitatory inputs to the striatum and the timing of the previous tic. A data-driven computational model of corticostriatal function closely replicated the temporal properties of tic generation and enabled the prediction of tic timing based on incoming cortical activity and tic history. These converging experimental and computational findings suggest a clear functional dichotomy within the corticostriatal network, pointing to disparate temporal (cortical) versus spatial (striatal) encoding. Thus, the abnormal striatal inhibition typical of Tourette syndrome and other tic disorders results in tics due to cortical activation of the abnormal striatal network.

Key words: animal model; basal ganglia; motor cortex; tic; Tourette syndrome

\section{Significance Statement}

The factors underlying the temporal properties of tics expressed in Tourette syndrome and other tic disorders have eluded clinicians and scientists for decades. In this study, we highlight the key role of corticostriatal activity in determining the timing of individual tics. We found that cortical activation determined the timing of tics but did not determine their form. A data-driven computational model of the corticostriatal network closely replicated the temporal properties of tic generation and enabled the prediction of tic timing based on incoming cortical activity and tic history. This study thus shows that, although tics originate in the striatum, their timing depends on the interplay between incoming excitatory corticostriatal inputs and the internal striatal state.

\section{Introduction}

The striatum contains feedforward and feedback GABAergic connections encompassing both projection and interneurons, forming an extensive inhibitory network. This unique structure prompted the hypothesis that the function of the basal ganglia (BG) in general and specifically of the striatum is to select a single

\footnotetext{
Received July 23, 2015; revised 0ct. 11, 2015; accepted 0ct. 18, 2015.

Author contributions: M.I. and I.B.-G. designed research; M.I. and I.B.-G. performed research; M.I. and I.B.-G. analyzed data; M.I. and I.B.-G. wrote the paper.

This study was supported in part by Israel Science Foundation Grant 743/13 and a Tourette Syndrome Association grant. We thank K. Belelovsky for her help with the histology, D. Cohen for her help with the EMG, and A. Korngreen for helpful comments.

The authors declare no competing financial interests.

Correspondence should be addressed to Izhar Bar-Gad, Bar-Ilan University, Gonda Brain Research Center, RamatGan 52900, Israel. E-mail: izhar.bar-gad@biu.ac.il.

DOI:10.1523/JNEUROSCI.2770-15.2015

Copyright $\odot 2015$ the authors $\quad 0270-6474 / 15 / 3516340-12 \$ 15.00 / 0$
}

action of a multitude of actions presented by massive convergent corticostriatal inputs. In this context, the GABAergic network inhibits all the actions presented by the cortex except for a single "selected" action that is released via the BG output by closing the loop back to the cortex (Mink, 1996). A breakdown of this GABAergic network is hypothesized to lead to abnormal action release by a subset of striatal neurons stemming from failed inhibition by the surrounding subpopulations representing the correctly selected actions (Mink, 2001; Albin and Mink, 2006). The abnormal release of an action associated with a specific somatotopic location within the striatum was recently attributed to the clinical symptom of motor tics (Bronfeld et al., 2013). Motor tics are repetitive, nonrhythmic, brief muscle contractions that appear as a common symptom in multiple disorders, most notably Tourette syndrome (TS; American Psychiatric Association, 2013). The pathophysiology of tics remains enigmatic but has been linked primarily to different abnormalities in the cortico- 
basal ganglia (CBG) pathway (Peterson et al., 2003; Singer and Minzer, 2003; Bloch et al., 2005; Kalanithi et al., 2005; Wang et al., 2011; Lerner et al., 2012) and most notably to abnormal inhibition within the striatum (Singer and Minzer, 2003; Kataoka et al., 2010).

Experimentally induced deficits in the striatal inhibitory network using local microinjections of different $\mathrm{GABA}_{\mathrm{A}}$ antagonists into the motor striatum in rodents (Marsden et al., 1975; Tarsy et al., 1978) and primates (Crossman et al., 1988) lead to the formation of simple motor tics that are associated with finely timed neuronal activation throughout the CBG pathway (McCairn et al., 2009, 2013; Bronfeld et al., 2011). Manipulation of $\mathrm{GABA}_{\mathrm{A}}$ receptor activation alters both feedforward, via fast spiking interneurons (FSIs), and feedback, direct via medium spiny neuron (MSN) collaterals or indirect via the globus pallidus (GP; Bennett and Bolam, 1994; Koós and Tepper, 1999; Mallet et al., 2012). While the tics initiate in the striatum and propagate through the CBG pathway, the role of the cortex within this loop is unclear. Blocking the information flow from the cortex to the striatum using cortical cooling or lesions (Muramatsu et al., 1990) was shown to eliminate observable tics but maintains tic-related neuronal activity in the striatum of the rat. Reduction of cortical input using cortical muscimol injections or striatal glutamate blockade (Pogorelov et al., 2015) reduced or even completely abolished visible tics in the mouse. These results suggest that the loss of corticostriatal input disrupts tics; however, the role of these afferents in tic generation is still unclear.

The transient modulation of striatal disinhibition leads to a prolonged period in which stereotypic tics are expressed at irregular intervals resembling those typical of TS (McCairn et al., 2009; Bronfeld et al., 2013). This period can be exploited to address the two key questions related to tic expression: "when" and "where" are tics expressed, i.e., what determines the time of an individual tic and the body region where this tic is expressed. The location of the focal disinhibition within the somatotopic organization of the dorsal striatum determines the body part expressing the tic, injection in the anterior striatum leads to forelimb tics, whereas injection in the posterior striatum leads to hindlimb tics, thus addressing the "where" question (Bronfeld et al., 2013). However, the neuronal activity underlying timing has been elusive, thus leaving the "when" question unanswered. The goal of this study was to address this question by examining the effect of corticostriatal activity and its interaction with the internal state of the striatal network on the timing of individual tics in the striatal disinhibition rat model.

\section{Materials and Methods}

Animals. Eleven adult rats (Long-Evans, nine females and two males) weighing $280 \pm 66 \mathrm{~g}$ (mean $\pm \mathrm{SD}$ ) were used in this study. The rats had access to food and water ad libitum and were maintained under a controlled $12 \mathrm{~h}$ light/dark cycle and temperature. All procedures were approved and supervised by the Institutional Animal Care and Use Committee and were in accordance with the National Institutes of Health Guide for the Care and Use of Laboratory Animals and the Bar-Ilan University Guidelines for the Use and Care of Laboratory Animals in Research. This study was approved by the National Committee for Experiments in Laboratory Animals at the Ministry of Health.

Surgery. Stimulation electrodes, a recording array, and an injection cannula were implanted in the right hemisphere of the rats. Two Narylene-coated stainless steel electrodes ( $145 \mu \mathrm{m}$ diameter; impedance, $\sim 25 \mathrm{~K} \Omega$; WeSense) were used for primary motor cortex (M1) stimulation. One electrode was implanted in the somatotopic region of the forelimb [anteroposterior (AP), $2.5 \mathrm{~mm}$; mediolateral (ML), $2.5 \mathrm{~mm}$; dorsoventral (DV), $1.75 \mathrm{~mm}$ ], and the second electrode was implanted in the hindlimb somatotopic region (AP, $-0.5 \mathrm{~mm}$; ML, $1.5 \mathrm{~mm}$; DV, 1.75 $\mathrm{mm}$; Paxinos and Watson, 2007). Custom-made movable bundles of 16 Formvar-insulated nichrome microwires (25 $\mu \mathrm{m}$ diameter; A-M Systems; Yael et al., 2013) were implanted in the striatum (AP, $0.25 \mathrm{~mm}$; ML, $3.75 \mathrm{~mm}$; DV, $4 \mathrm{~mm}$ ) or the GP (AP, $-0.84 \mathrm{~mm}$; ML, $3 \mathrm{~mm}$; DV, 4.6 $\mathrm{mm}$ ) for neuronal recording. A guide cannula (stainless steel 25 gauge tube) was implanted to enable microinjections into the anterior striatum (injection target: AP, $1.4 \mathrm{~mm}$; ML, $2.5 \mathrm{~mm}$; DV, $4.6 \mathrm{~mm}$ ). This injection region was shown previously to be in the forelimb somatotopic region of the striatum (Bronfeld et al., 2013). After implantation, the cannula was sealed temporarily with a dummy (stainless steel 30 gauge wire). A pair of Teflon-coated silver wires (bare diameter, 0.003 inch; A-M Systems) were implanted in the left triceps muscle of some rats for electromyogram (EMG) recording purposes.

Experimental sessions. The experiments began after a recovery period of at least $7 \mathrm{~d}$ after the surgery. During the experimental sessions, the neurophysiological data were recorded continuously while the animal was moving freely in the recording cage. The electrode signal was amplified $(200 \times)$, wide bandpass filtered $(0.5-10,000 \mathrm{~Hz}$, four-pole Butterworth filter), and sampled continuously at $44 \mathrm{kHz}$ (Alpha-Lab SNR; Alpha-Omega Engineering). Movement was assessed using EMG recordings (20× amplification, $2.75 \mathrm{kHz}$ sampling) and a two-camera video acquisition system ( 60 frames/s; NorPix).

At the beginning of each experimental day, the motor threshold (MT) of the forelimb and the hindlimb regions in M1 were set for either high or low stimulation frequencies (see Fig. 2B). Stimulation in the forelimb region occasionally induced muscle contractions of the neck in addition to the forelimb. The absolute stimulation amplitudes used for the experiment were set as a function of the MT. Stimulation bursts (20/25/50 stimuli were used in $34 / 1 / 3$ sessions of 38 sessions, respectively, $176 \mathrm{~Hz}$, biphasic, 250 $\mu$ s per phase) were delivered using a backpack-based wireless stimulator (Deuteron Technologies) that was worn on the rats' back in a custom-made backpack. Each session contained 50 repetitions of the stimulation bursts with random interburst intervals ( $1-5 \mathrm{~s}$, mean of $3 \mathrm{~s}$ ). Sessions examining varied amplitudes included 35 repetitions of the stimulation bursts ( 50 stimuli, $176 \mathrm{~Hz}$, biphasic, $250 \mu \mathrm{s} /$ phase) per amplitude.

Bicuculline methiodide (Sigma-Aldrich) was dissolved in artificial CSF to a final concentration of $1 \mu \mathrm{g} / \mu \mathrm{l}$. After stimulation in the naive state, microinjections of $0.35 \mu \mathrm{l}$ (in $8 \%$ of the injections, a volume of up to $0.7 \mu \mathrm{l}$ was used) bicuculline were pressure injected through an injection cannula (stainless steel 30 gauge tube, protruding $2 \mathrm{~mm}$ from the guide cannula) at a rate of $0.35 \mu \mathrm{l} / \mathrm{min}$ (NE-1000; New Era Pump Systems). Bicuculline injection within this target was shown recently to lead to tics primarily in the forelimb area (Bronfeld et al., 2013). Previous studies demonstrated that comparable bicuculline injection spreads in an ellipsoid with an approximate diameter of $<1 \mathrm{~mm}$ around the injection site (Yoshida et al., 1991).

Data preprocessing and analysis. The recorded data were pre-processed offline to extract the basic signals, local field potentials (LFPs), and single-unit spike trains, used for additional analysis. Stimulation artifacts were removed using SARGE (Erez et al., 2010) generating artifact-free data streams, and the signals were sorted offline (Offline Sorter, version 2.8.8; Plexon) into multiple single-unit spike trains. Neurons presenting unstable waveforms or firing patterns were excluded from the database. The remaining neurons were divided into three different neuron types (MSN, FSI, and GP) according to their location, firing rate, firing pattern, and waveform shape and duration (Benhamou et al., 2012; Yael et al., 2013). Tonically active neurons and other striatal interneurons were excluded from the database. All additional analyses were performed using custom-written MATLAB code (V2012B; MathWorks).

The LFP signal was obtained from the recorded data using a low-pass filter ( $40 \mathrm{~Hz}$, four-pole zero-phase forward-backward Butterworth filter). LFP spikes were detected using a threshold-crossing method on the LFP signal (see Fig. 2A). Tic onset time was calculated by removing twice the time from the minima/maxima to the point of half height. Noisy periods were detected and removed from the analysis. The EMG signal was defined as the differential signal of the two wires. The signal was then bandpass filtered $(5-300 \mathrm{~Hz}$, four-pole zero-phase forward-backward Butterworth filter), and specific noise frequencies were removed using notch filters. 
A

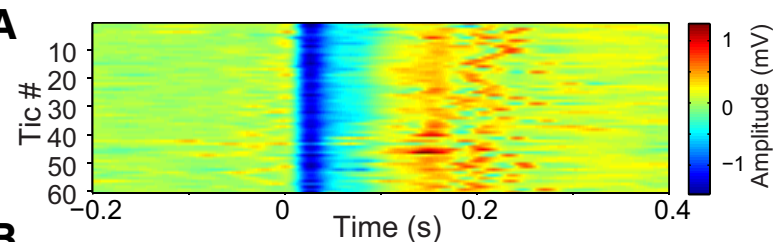

B
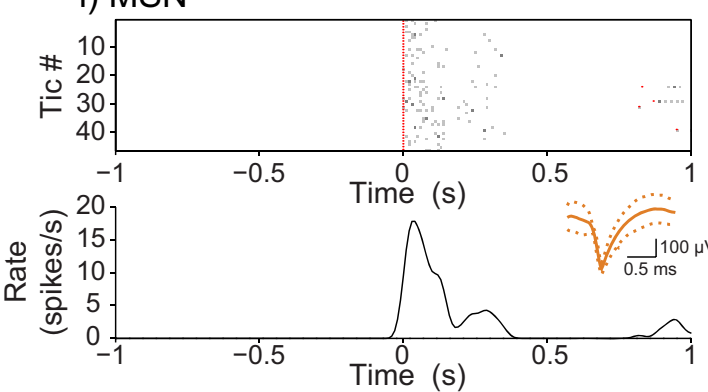

iii) GP
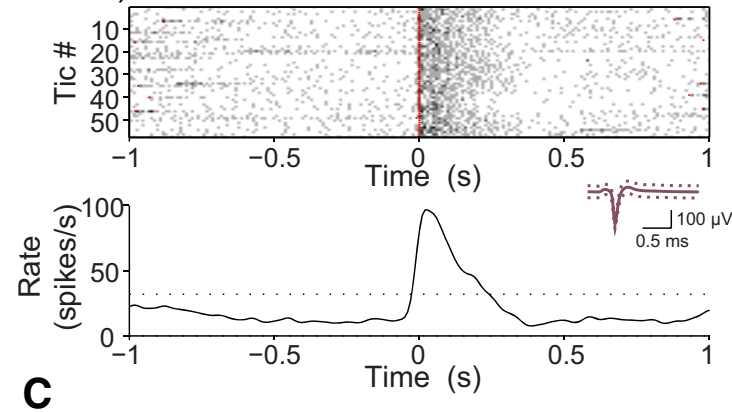

C

\section{MSN}

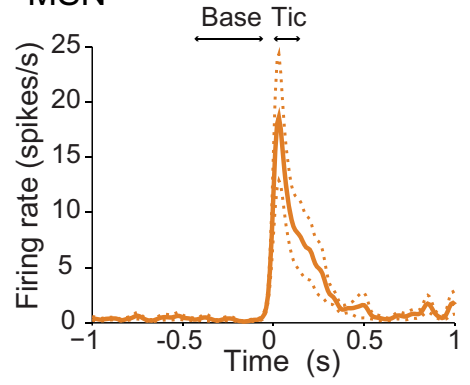

D

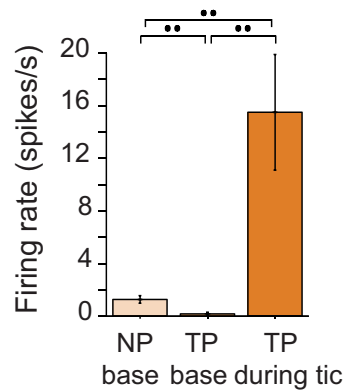

FSI
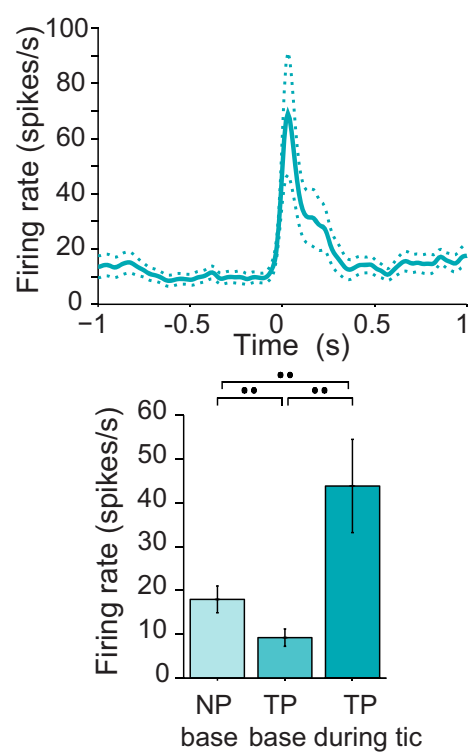

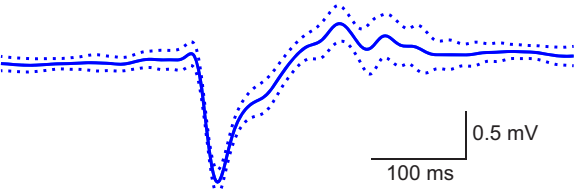

ii) FSI
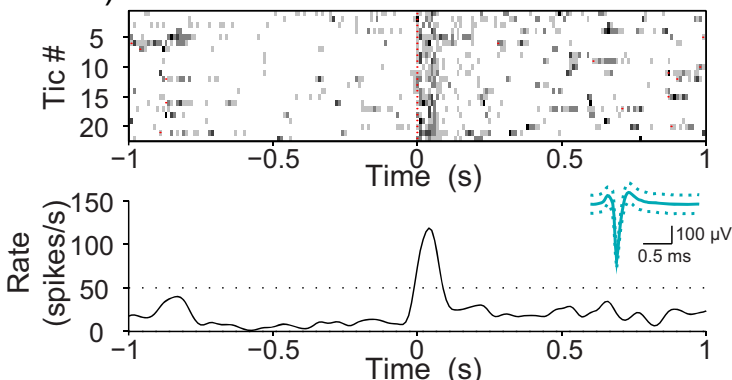

IV) GP

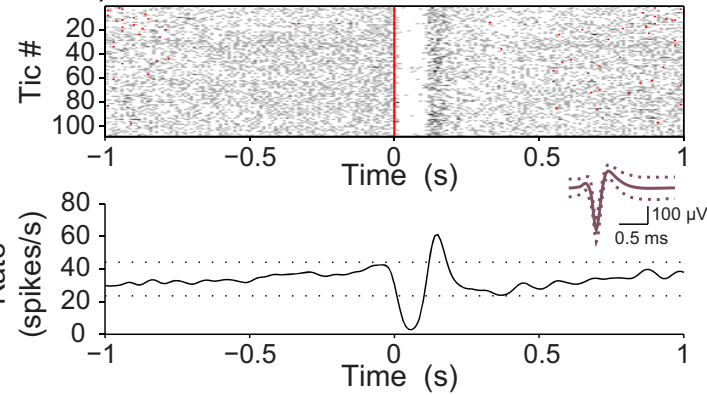

GP
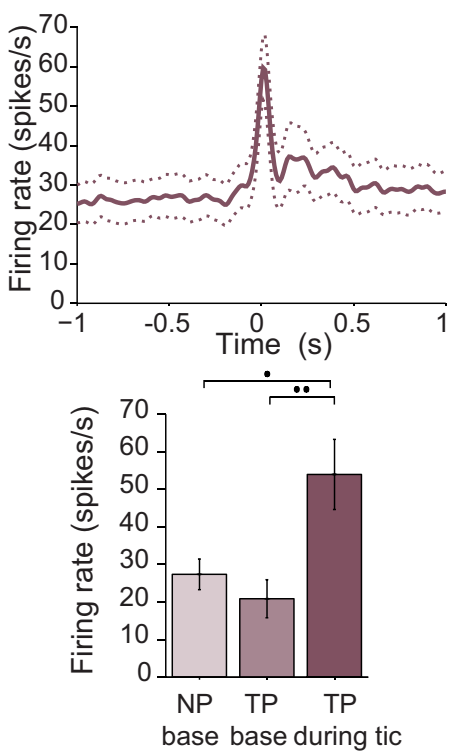

Figure 1. Tic-related neuronal activity. $\boldsymbol{A}$, Peri-tic LFP spikes (left) and the mean LFP spike shape (bold) \pm 1 SD (dotted) (right). $\boldsymbol{B}$, Peri-tic single-neuron raster (top) and peri-tic histogram (bottom), for example, MSN (i), FSI (ii), and GP neurons (iii, iv). Red dots, tic times. Black dotted lines in the histograms are the upper and lower $99 \%$ confidence intervals. The mean waveform of the individual neurons (solid) \pm 1 SD (dotted) appear in the inset. C, Mean peri-tic response of the neuronal population (bold) \pm 1 SEM (dotted). D, Mean firing rate of MSNs (left), FSls (middle), and GP neurons (right) during the NP (NP base), the TP (TP base), and during the $100 \mathrm{~ms}$ after the tics (TP during tic) \pm 1 SEM. ${ }^{*} p<0.05$, $^{* *} p<0.01$.

Tic-related neuronal activity was assessed using peri-tic time histograms (PTTHs), using the individual tic times as a point process. The PTTH was calculated using 0.01 s bins and was smoothed with a Gaussian window (SD of $20 \mathrm{~ms}$ ). Significant tic-related neuronal activity was determined by calculating the two-tailed confidence intervals based on the PTTH tail distribution ( 1 to $0.1 \mathrm{~s}$ before tic onset) with Bonferroni's correction. Changes in the overall neuronal firing rate were assessed by comparing the baseline firing rate of each neuron during the normal period (NP) with the baseline firing rate during the tic period (TP; calculated over the 0.5 to $0.1 \mathrm{~s}$ interval before tic onset) and during tic occurrence time ( 0 to $0.1 \mathrm{~s}$ after tic onset) using paired-sample $t$ tests between each pair of groups. The mean firing rate of the population was calculated using only the neurons that were recorded and maintained throughout both the normal and the TPs (MSN, 19 of 22; FSI, 19 of 22; GP, 17 of 23). The relationship between stimulation and behavior was assessed by using peristimulus tic histograms with $0.01 \mathrm{~s}$ bins. To calcu- 


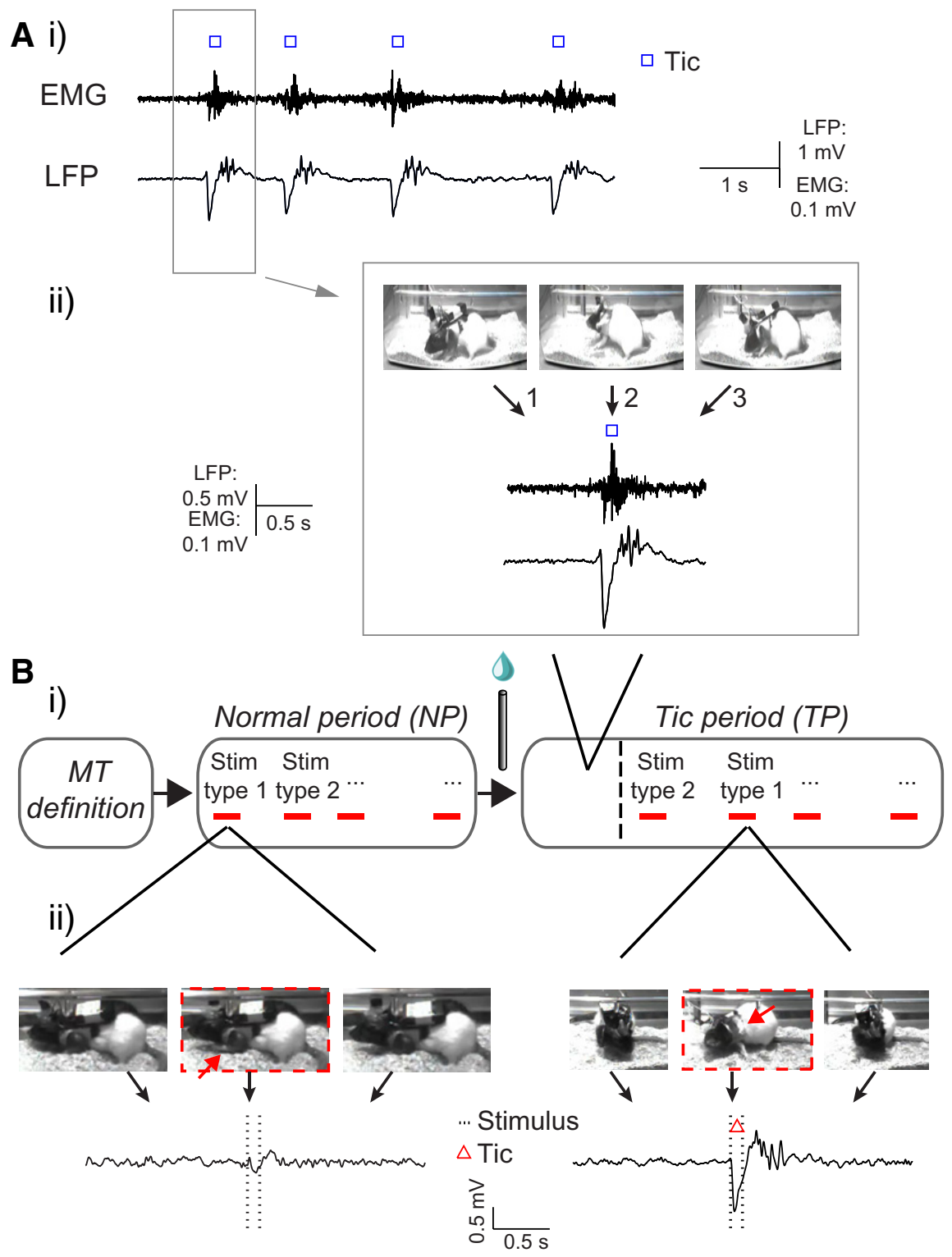

Figure 2. Experimental session. $\boldsymbol{A}$, An example of tic expression after bicuculline injection. $\boldsymbol{i}$, Simultaneously recorded EMG (top) and LFP (bottom) activity during tic expression. ii, Individual tic identification using video (top), EMG (middle), and the associated LFP spikes (bottom). Video frames 1-3 represent pre tic, during tic, and post tic time, respectively. $\boldsymbol{B}, \boldsymbol{i}$, A schema of an experimental session including MT definition, followed by cortical stimulation during the NP and TP separated by the bicuculline injection. ii, An example of the stimulation effect on behavior (video, top) and LFP (bottom) during the NP (left) and the TP (right).

late the stimulation success percentage in inducing tics, unsuccessful stimuli that were immediately $(500 \mathrm{~ms}$ ) preceded by a tic were removed from the total number of stimuli in the calculation.

The correlations between the shape of the stimulation-induced (SI) and spontaneously occurring (SO) tic-related LFP spikes and neuronal activity were assessed by calculating the Pearson's product-moment correlation coefficient (PCC). Correlations were also calculated within the SO tic group and the SI tic group separately by randomly dividing each group into two equal groups. Neurons that did not significantly change their activity during spontaneous tics were excluded from the tic-related neuronal activity correlation calculation (MSN, 1 of 22; FSI, 3 of 22; GP, 0 of 23 ).

Throughout Results, all the numbers are shown as mean \pm SD, unless stated otherwise.

Histology. At the end of the experimental sessions, the rats were anesthetized, and the location of the electrode tips were marked using microlesions $(-100 \mu \mathrm{A}, 5-20 \mathrm{~s})$. The rats were then perfused transcardially, and the brains were stored in a sucrose $(30 \%)$ and paraformaldehyde
(10\%) solution. After $>48 \mathrm{~h}$, the brains were frozen and cut into slices of $50 \mu \mathrm{m}$ in the coronal plane. The slices were stained using cresyl violet to identify the anatomical structures and verify the locations of the injection, stimulations, and the recording electrodes.

\section{Results}

Bicuculline injection leads to the formation of tics and associated neuronal activity

Injection of bicuculline into the anterior motor region of the striatum led to a period of ongoing nonregular focal tics in the forelimb area contralateral to the injection side (see Movie 1), tic period (TP), similar to those described previously (Bronfeld et al., 2013). Tics were characterized as focal, repetitive, brief muscle contractions that were identified using high-speed video recordings and subcutaneous EMG. In 41 experimental sessions performed on 11 animals, the tics began $1.8 \pm 0.9 \mathrm{~min}$ after the injection termination and lasted for $55.1 \pm 18.5 \mathrm{~min}$. As shown previously in primates (McCairn et al., 2009; Bronfeld et al., 2011), individual tics were associated with stereotypic low-frequency changes in the local field potential (LFP) ("LFP spike") that was coherent across the electrodes (Figs. 1A, 2A).

We recorded the activity of 67 neurons from five animals during 15 experimental sessions simultaneously with the behavioral and LFP recordings. The neurons were identified as MSNs $(n=22)$ and FSIs $(n=22)$ in the striatum and GP neurons $(n=23)$ based on their characteristic waveform, firing rate, and pattern. The vast majority of the neurons (MSN, 21 of $22,95 \%$; FSI, 19 of $22,86 \%$; GP, 23 of 23 , $100 \%$ ) displayed a significant change in activity time locked to the tics (Fig. $1 B$ ). In line with previous results from primates (McCairn et al., 2009), several different response types were found (Fig. $1 B$ ). All of the significantly modulated MSNs (21 of $21,100 \%$ ) displayed a short burst of activity during the tic but were almost or totally quiescent at all other times (Fig. 1Bi). All the significantly modulated FSIs (19 of 19, $100 \%$ ) displayed an increase in activity during the tic that was followed by a decrease in activity in a minority of the neurons (4 of 19, 21\%; Fig. 1Bii). The GP neurons demonstrated heterogeneous response patterns that were clustered into four groups: (1) excitation only (Fig. 1Biii); (2) inhibition only; (3) excitation followed by inhibition; and (4) inhibition followed by excitation (Fig. 1Biv). Approximately half of the recorded GP neurons (12 of $23,52 \%$ ) displayed a single excitation phase, whereas the rest were approximately equally divided between the other groups. The mean response of the populations for all the recorded neuronal types to the tics was a short increase in activity around the time of tic onset (Fig. 1C). In contrast to this finely timed ticrelated increased activity, a significant decrease was found in the 
baseline firing rate of the striatal neurons during the TP compared with the preceding normal period (NP) (Fig. 1D).

\section{Cortical stimulation leads to tic formation}

We tested the effect of cortical microstimulation on behavior and neuronal activity before the bicuculline injection (NP) and during the spontaneous tic expression period after the injection (TP; Fig. 2B). Cortical stimulation, a short burst of high-frequency stimulus pulses, was applied at random intervals to the somatotopic region of the primary motor cortex (M1) corresponding to the tic somatotopic expression area (i.e., the forelimb). Initially, the motor threshold (MT) was established during the NP of each session and was defined as the minimal stimulation amplitude required for eliciting movement. Forelimb suprathreshold $(>\mathrm{MT})$ cortical stimulations were administered in 38 experimental sessions. Stimulation during the TP led to the expression of a single tic during the stimulation burst and the formation of the associated LFP spike (Fig. $2 B$, right). In contrast, the same stimulation during the NP elicited a small movement that was unrelated to the tics and was not associated with an LFP spike (Fig. 2B, left). Tics that appeared within a short window from the stimulation burst initiation $(200 \mathrm{~ms})$ were defined as stimulation-induced (SI) tics, and all other tics during the TP were defined as spontaneously occurring (SO) tics. The SI tics were intermingled with the continued occurrence of SO tics throughout the TP (Fig. 3A). Cortical stimulation elicited SI tics locked to the stimulation onset with high fidelity: $97.8 \pm 3.6 \%$ of the stimuli that were not immediately $(500 \mathrm{~ms})$ preceded by an $\mathrm{SO}$ tic resulted in an SI tic (Fig. $3 B, C$ ). The SO tics and the SI tics appeared qualitatively identical after video-based analysis (see Movie 1). We quantitatively assessed the similarity between SO and SI tics by examining the similarity of their neuronal encoding on the macroscale and microscale. On the macroscale, no significant differences were found between the shapes of the LFP spikes related to SI and SO tics (Fig. 4Ai). The PCC between the LFP shapes of SO and SI tics in each session was high (0.95 \pm 0.005 , mean \pm SEM) and not statistically different from the PCC across SO or SI tics within the session (SI-SI PCC, $0.96 \pm 0.005$; SO-SO PCC, $0.95 \pm 0.006$, mean \pm SEM; Fig. $4 B$ ). On the microscale, SI tics were associated with changes in single-neuron activity that were similar to SO tic-related activity (Fig. 4Aii). Correlations between the PTTH of SO and SI tics of each neuron that responded to SO tics revealed similar firing rate modulations around tic onset time (PCC; MSN, $0.79 \pm 0.06$; FSI, $0.84 \pm 0.03$; $\mathrm{GP}, 0.85 \pm 0.025$, mean $\pm \mathrm{SEM})$ that was comparable with the variability of the response within the groups (SI-SI PCC: MSN, $0.79 \pm 0.06$; FSI, $0.86 \pm 0.03$; GP, $0.85 \pm 0.04$; SO-SO PCC: MSN, $0.79 \pm 0.05$; FSI, $0.81 \pm 0.06$; GP, $0.8 \pm 0.03$, mean \pm SEM; Fig. $4 B$ ). Thus, in addition to the observed behavioral similarity of SO and SI tics, their neural correlates did not appear to be different on the single-neuron and large-population (LFP) levels. Furthermore, the generation of a tic led to an equal reset process of the striatal network regardless of the factors leading to it. Thus, when elicited, the mean time interval between an SI tic and the subsequent $\mathrm{SO}$ tic was similar to the mean time interval between two adjacent SO tics (calculated on sessions containing at least five pairs from each group; Fig. 4C).

Tics are stereotypic regardless of cortical input properties We tested the effect of cortical activation properties on the tic and its neuronal encoding. Different values of the stimulation amplitude, frequency and the somatotopic location within M1 of the
A $\quad \cdots$ Stimulus

口 SO tic

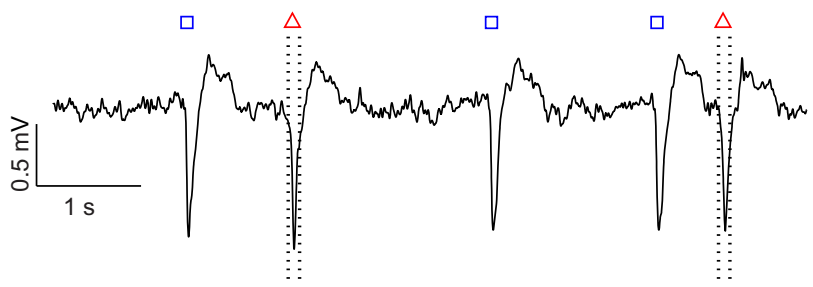

B

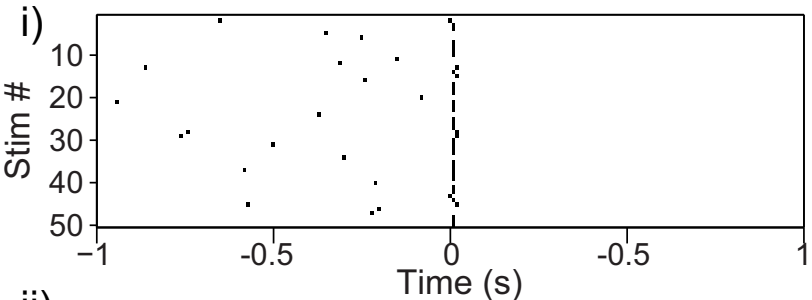

ii)

Time (s)
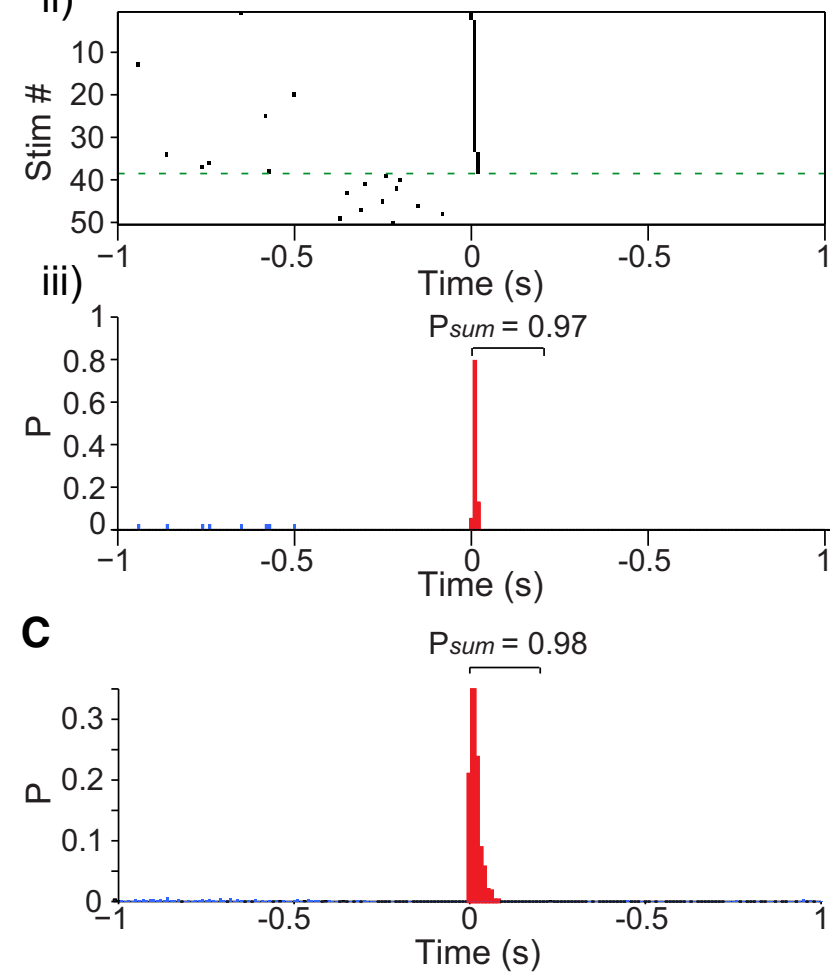

Figure 3. Cortical stimulation induces tics. $A$, Short LFP trace during the TP including both $S 0$ and SI tics. $\boldsymbol{B}$, Single session tic formation after stimulation: peristimulus tic raster (i) and the same raster sorted according to the latency between stimulation onset and the induced tic (ii). The green dotted line separates trials in which a tic appeared $<0.5$ s before the stimulation. iii, Peristimulus tic histogram of the trials above the green line. The colored bars represent $\mathrm{SI}$ (red; up to $200 \mathrm{~ms}$ after stimulation onset) and $S O$ (blue) tics. $P_{\text {sum }}$ denotes the total probability for a tic within the defined window $\left(P_{\text {sum }}=\sum_{t=0}^{200} P\right)$. C, Population peristimulus tic histogram.

stimulating electrode (Fig. 5A) all induced SI tics that were identical to the SO tics (Fig. 5). Specifically, electrodes located in two separate somatotopic areas in M1 delivered the stimulation to the forelimb, which is also the location of the tic, and the hindlimb. High-frequency stimulation $(176 \mathrm{~Hz})$ of the hindlimb region was administered in 12 sessions using suprathreshold stimulation 
A i)

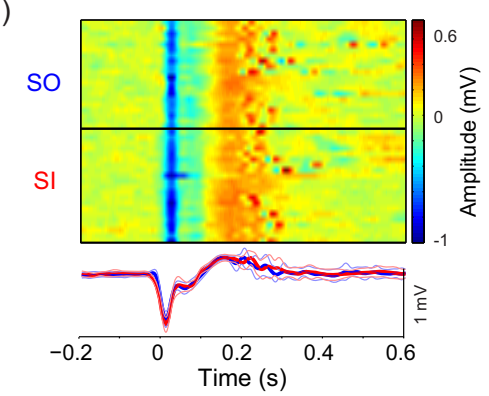

B

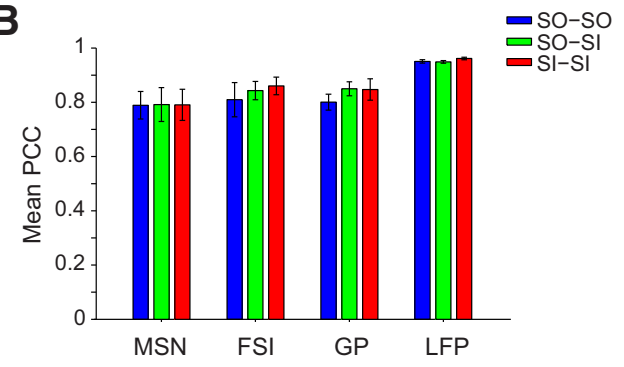

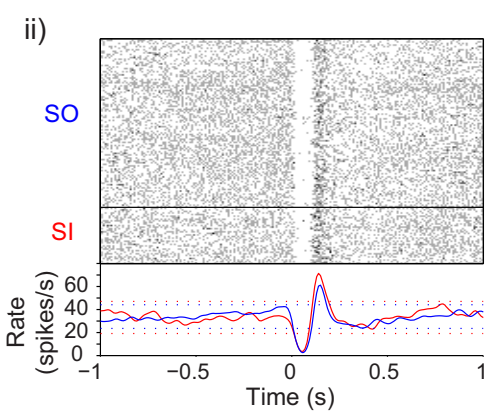

C

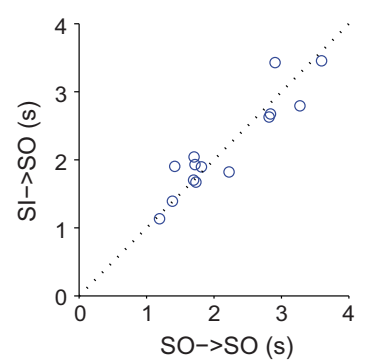

Figure 4. Similarity of SO and SI tics. $\boldsymbol{A}, \boldsymbol{i}$, Multiple trials (top) and mean (bottom) SO (blue) and SI (red) LFP spike shapes during a single session. The solid black line segregates $\mathrm{SO}$ and $\mathrm{SI}$ tics recorded during the same stimulation session. Bold lines, Mean shape; thin lines, single SD. ii, Peri-tic raster (top) and histogram (bottom) of SO (blue) and SI (red) tic-related neuronal activity of a single GP neuron. Dotted lines in the histogram are the upper and lower $99 \%$ confidence intervals. $\boldsymbol{B}$, Mean population comparison of PCC of neuronal activity and LFP shapes between SI and SO tic (green) and within SO (blue) or SI (red) tics \pm 1 SEM. C, The mean time interval for each session between two adjacent $\mathrm{SO}$ tics compared with the mean time interval between an $\mathrm{SI}$ tic and the subsequent So tic.

amplitude. During the NP, different stimulation locations led to the movement of only the limb associated with the corresponding stimulation somatotopic location. However, during the TP, the location of the stimulating electrode did not change the expression of the SI tic that was always confined to the body part corresponding to the location of the bicuculline injection (hindlimb stimulation success rate in eliciting forelimb tics, $99.1 \pm 1.8 \%$ ) and was similar in its encoding to the SO tics (LFP spike PCC between SO and SI tics for hindlimb stimulation, $0.86 \pm 0.08$; Fig. $5 B-D$; see Movie 2). The stimulation amplitude, relative to the $\mathrm{MT}$ required to elicit a movement, was manipulated in 20 sessions between suprathreshold (132.9 $\pm 25.5 \%$ of MT) stimulation and subthreshold $(68.9 \pm 14.9 \%$ of MT) stimulation. During the TP, both suprathreshold and subthreshold stimulation led to the formation of identical tics (LFP spike PCC between SO and SI tics for subthreshold stimulation, $0.93 \pm 0.06$; Fig. $5 B-D)$. Finally, the stimulation frequency was alternated between high frequency $(176 \mathrm{~Hz})$ and low frequency $(44 \mathrm{~Hz})$ in 17 sessions while maintaining the same number (20) of stimulation pulses. In both frequencies, we used suprathreshold stimulation amplitudes that conformed specifically to the MT defined for each frequency. During the TP, both stimulation frequencies led to similar SI tics (LFP spike PCC between SO and SI tics for $44 \mathrm{~Hz}$, $0.89 \pm 0.05$; Fig. $5 B-D$ ). Thus, SI tics appear as stereotypic discrete events whose expression is dependent on cortical activity, but its form is invariant to the specific nature of this input.

\section{Internal and external factors affecting tic timing}

The individual tics elicited using the stimulation parameters described above did not differ in their shape and amplitude; however, the number of stimulation pulses required to elicit the SI tic and the probability of tic generation was a function of the amplitude of each pulse (Fig. $6 A, B$ ). We varied the stimulation ampli- tude in each session, during 10 sessions. The probability of the formation of an SI tic increased with the amplitude of the stimulation in a sigmoid manner $\left(r^{2}=\right.$ 0.97, $p<0.01$ ) depending on the amplitude of the stimulation relative to the MT (Fig. 6C). Stimulation below $40 \%$ of MT elicited tics with a probability of $<50 \%$, and lower amplitudes ( $<25 \%$ of MT) did not yield SI tics above chance level. In addition, the latency between the stimulation burst beginning and the tic initiation decreased with the stimulation amplitude (Fig. 6B) in a truncated exponential manner $\left(r^{2}=0.93, p<0.01\right.$; Fig. $\left.6 D\right)$, i.e., higher stimulation amplitude led to rapid formation of tics, indicating that tic generation is a cumulative process of cortical input summation.

The occurrence of tics during the time preceding the stimulation affected the probability of the formation of an SI tic and its latency, i.e., the number of pulses required for tic generation. A tic occurring immediately before the stimulation, 0-250 ms before the stimulation onset, prevented the formation of an SI tic (tic release $p \leq 0.1$ ) in a manner resembling the refractory period of a neuron. This period was termed the absolute tic refractory (ATR) period (Fig. $7 A, B$ ). A tic occurring within a time window preceding the ATR period, 251-750 ms before the stimulation onset, was termed the relative tic refractory (RTR) period and led to a decrease in the probability of tic generation (tic release $0.1<$ $p<0.9$ ) and an increase in the number of stimulation pulses required for its generation in a gradual manner (Fig. $7 B$ ). The probability of the formation of an SI tic increased as a function of the time difference from the stimulation onset to the time of the last tic before it $(\Delta t)$ in a sigmoidal manner $\left(r^{2}=0.97, p<0.01\right.$; Fig. $7 C$ ). The number of stimulation pulses required to elicit an SI tic decreased as a function of $\Delta t$ in an exponential manner $\left(r^{2}=0.84, p<0.01\right.$; Fig. $\left.7 D\right)$.

\section{Tic generation model}

Our data suggest that, during the TP, the striatal network generates tics as a function of two interacting factors: (1) cortical input to the striatum; and (2) the timing of the previous tic. These two factors are reminiscent of the properties of the formation of spikes by individual neurons, although tics are generated by the striatal network over timescales that are several orders of magnitude longer, i.e., seconds compared with milliseconds. Based on this observation, we generated a phenomenological computational model that embodies an analytical description of the tic-generation mechanism. The model, which we term the tic generation model (TGM), is based on two kernels that are used for scaling the factors (cortical input and striatal feedback). The model is a simplified version of the simplified spike response model $\left(\mathrm{SRM}_{0}\right)$ that has been used extensively to model neuronal activity (Gerstner, 1995). In the TGM model, incoming cortical inputs lead to an increased activation of the striatal network, $A(t)$. These inputs either arrive in a Poissonian manner $\left(t_{c}\right.$, cortical activity; mean rate, $\frac{\sum_{i=1}^{n} \int_{0}^{T} \rho_{C_{i}}(t) d t}{T}$ 
$=100 \mathrm{~Hz}$, where $n$ is the number of cortical neurons projecting to the disinhibited area of the striatum and $\rho_{c}$ is the spike train of each neuron) or as a coactivation of multiple $(S)$ inputs $\left(t_{s}\right.$, SI activity; mean rate, $\frac{\int_{0}^{T} \rho_{s}(t) d t}{T}=$ $0.47 \mathrm{~Hz}$ ) and are multiplied by the $\alpha$ function response kernel $(C)$ :

$$
C(x)=x \cdot e^{-\left(x / \tau_{c}\right)} \cdot A_{C},
$$

where $\tau_{c}$ is the time constant of the cortical input, and $A_{c}$ is its amplitude. The previous tic $\left(t^{\prime}\right)$ reduces the activation for short periods after the tic following multiplication with the exponential function reset kernel $(R)$ :

$$
R(x)=-e^{-\frac{x}{\tau_{R}}} \cdot A_{R},
$$

where $\tau_{R}$ is the time constant of the striatal network, and $A_{R}$ is the amplitude of its effect. Whenever the activation level crosses a fixed threshold, a tic is generated (Fig. 8A):

$$
\begin{aligned}
A(t)=R\left(t-t^{\prime}\right)+ & \sum_{t_{c}} C\left(t-t_{c}\right) \\
& +S \cdot \sum_{t_{s}} C\left(t-t_{s}\right) .
\end{aligned}
$$

When no stimulation is applied, the model displays non-regular SO tics expressed at a frequency comparable with the one observed in the animals. The addition of stimulation results in SI tics during the stimulation burst (Fig. 8B). Finally, stimulation in close temporal proximity to a previous tic fails to induce a new tic (Fig. 8B, marked by an arrow). The probability of the generation of an SI tic closely resembles the one observed experimentally (Fig. 7C) with both ATR and RTR and a sigmoid behavior of tic probability $\left(r^{2}=0.99, p<0.01\right.$; Fig. $\left.8 C\right)$. Modeling the short stimulation bursts used experimentally revealed that tics may be evoked during the relative refractory period via accumulation of stimuli (Fig. 8D). Similar to experimental observations, the time from the start of stimulation to the tic initiation decreased with increasing time between the stimulus and the time of the tic before it, in a truncated exponential manner $\left(r^{2}=0.84, p<0.01\right.$; Fig. $8 D)$.

\section{Discussion}

The neuronal mechanism that determines the timing of individual tics is one of the key open questions in TS and other tic disorders. In this study, we addressed this question by exploring the effect of cortical excitation on tic generation in the striatal disinhibition animal model of motor tics. Cortical stimulation induced tics that were identical to the spontaneously occurring tics, regardless of the cortical input type, and led to similar ticrelated activity changes in both striatal and pallidal neurons. The

\section{LFP spikes}

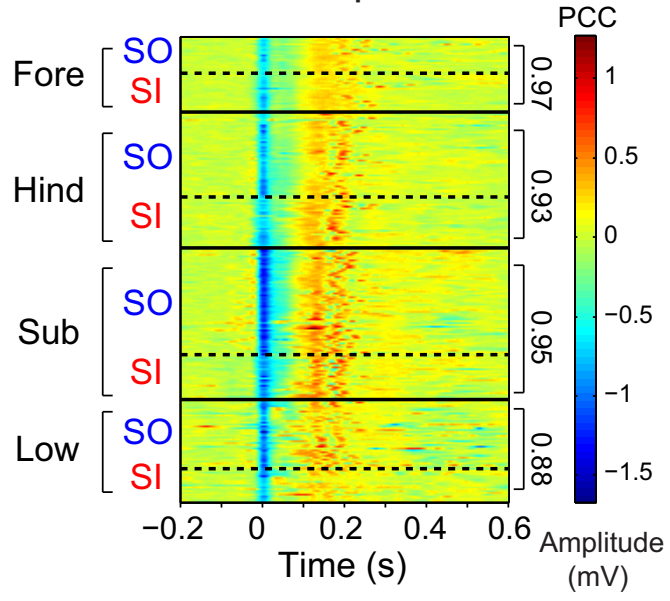

B MSN neuron

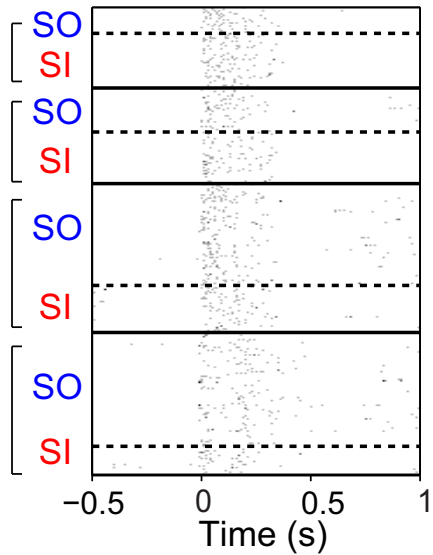

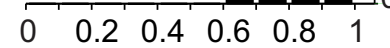

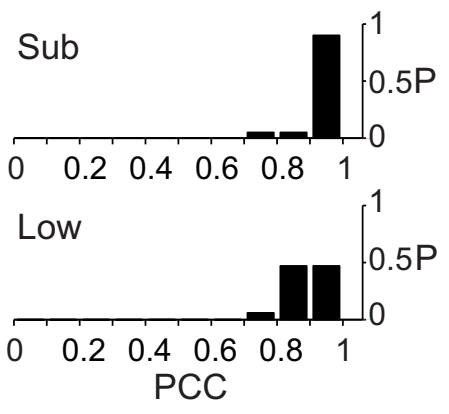

Figure 5. Tics are stereotypic. The neuronal representation of the tic is similar regardless of the stimulation parameters. $\boldsymbol{A}$, The stimulation parameters used in this study, varying the stimulation amplitude (suprathreshold/subthreshold), frequency (high/ low), and somatotopic location (forelimb/hindlimb). Peri-tic raster of SO and SI tic-related neuronal activity of a single MSN (B) and same stimulation session. The solid black lines differentiate different conditions. D, Population comparison of the PCC of the LFP shapes between $\mathrm{SI}$ and $\mathrm{SO}$ tics during different stimulation parameters.

effect of corticostriatal input on tic timing was cumulative and was affected by the amplitude and number of stimulation pulses. The generation of tics was limited by absolute and partial tic refractory periods that were derived from an internal striatal state. Thus, the summation of cortical input and the timing of the previous tic interact to determine the precise time of the next tic expression. This notion was supported by a simplified computational model of tic formation, which enabled the prediction of tic timing based on incoming cortical activity and tic history, thus closely replicating the experimental results.

The involvement of the cortex in tic generation has been studied in the striatal disinhibition model using temporary and irreversible lesions of the motor cortex (Muramatsu et al., 1990). After the lesions, the tics disappeared; however, the associated LFP spikes persisted and maintained their stereotypic shape while their frequency decreased. Our findings are in line with these results, because they demonstrate that the cortex drives the time of the tics but not their form of expression. Thus, a reduction in cortical input, such as after a lesion of parts of the cortex, is expected to result in an increased 
A i)
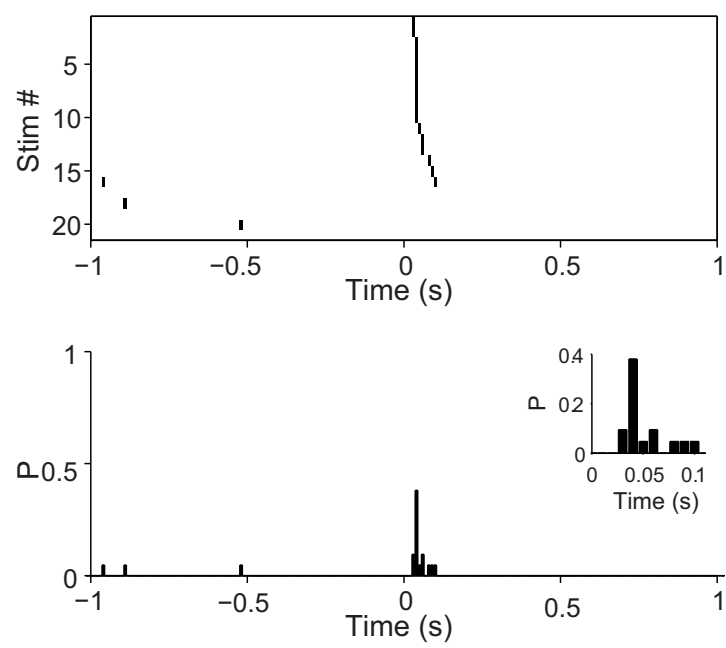

ii)

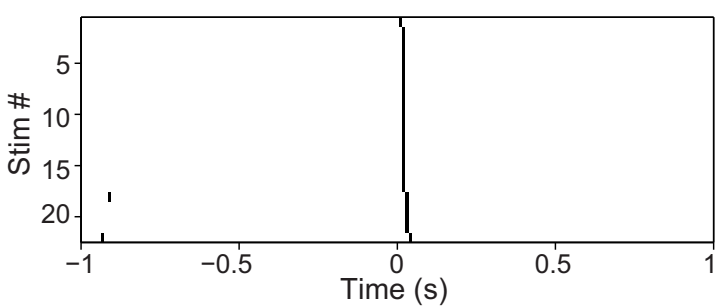

B
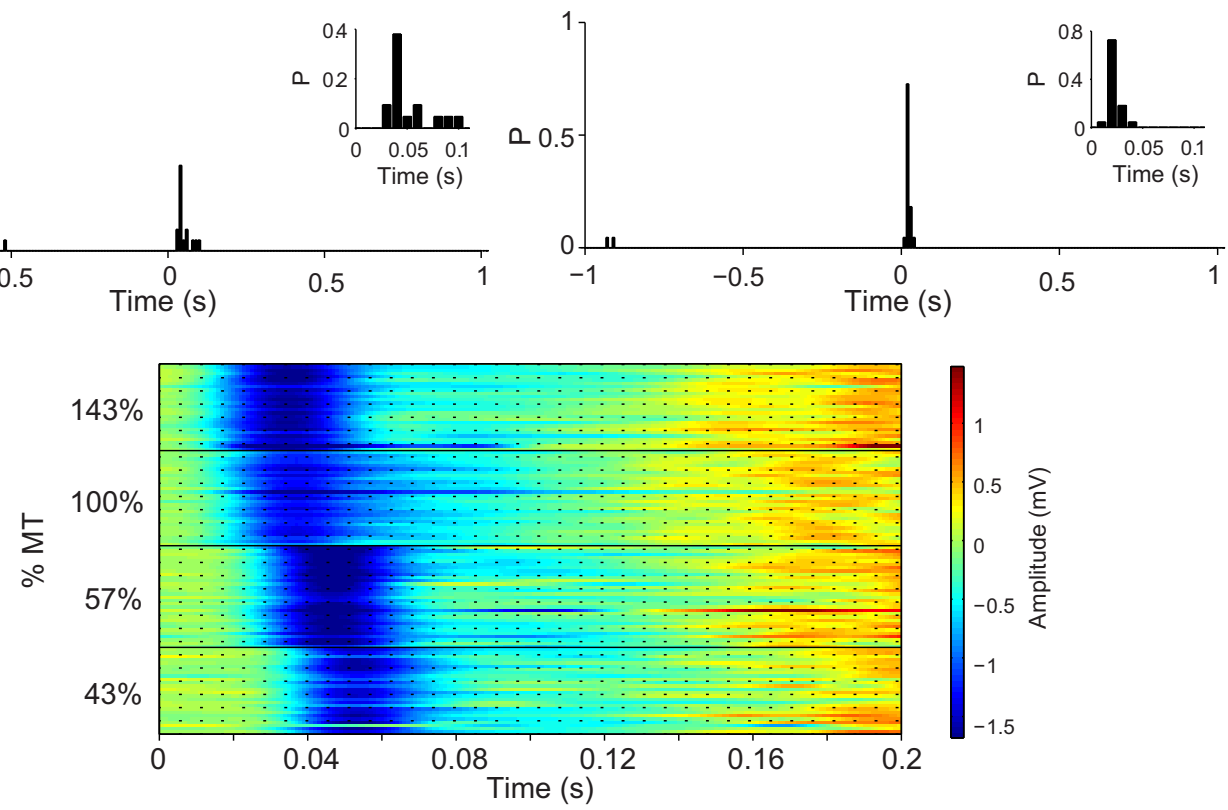

C
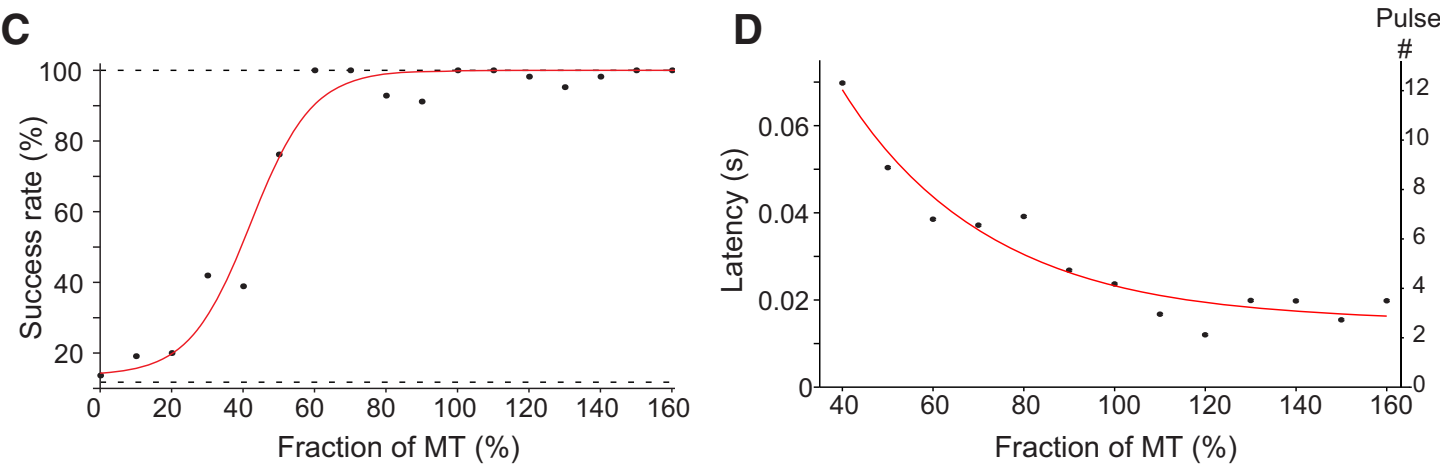

Figure 6. Tic generation is a cumulative process. The probability and latency of tic generation are dependent on stimulation amplitude. $A$, Example of sorted peristimulus tic raster (top) and peristimulus tic histogram (bottom) of $\mathrm{SI}$ tics in response to subthreshold stimulation (50\% of MT; left) and suprathreshold stimulation (117\% of MT; right). The insets show a higher-resolution view of the time immediately after the stimulation. B, SI induced LFP spikes at four different amplitudes (143, 100, 57, and 43\% of MT). Dotted black lines, Individual stimulation pulses. C, The median fraction of stimulation bursts resulting in an $\mathrm{SI}$ tic as a function of the stimulation amplitude (bin size, $10 \%$ MT). Red line, Sigmoid curve fitting. D, The mean time differences between stimulation onset and tic formation as a function of the stimulation amplitude (bin size, 10\% MT). Red line, Exponential curve fitting.

inter-tic interval. Injection of glutamate antagonist into the striatum eliminated the tics, indicating that glutamatergic inputs are essential for tic generation and tics do not result from spontaneous isolated striatal activity (Pogorelov et al., 2015).

The existence and duration (several hundred milliseconds) of the striatal network refractory periods may be attributed to two potentially complementary underlying neural mechanisms: (1) external, cortical-based inhibition; or (2) internal, striatal-based inhibition. Cortical stimulation performed on both human subjects and primates resulted in short-term cortical excitation, followed by a prolonged inhibition period
(Krnjevic et al., 1966; Inghilleri et al., 1993; Tischler et al., 2012). This period limits the ability of the cortex to be reexcited shortly after the preceding excitation (Kujirai et al., 1993; Werhahn et al., 1999). Thus, no cortical excitation can occur after either SO or SI tic manifestation. The tic refractory period may also be attributed to activation of presynaptic and/or postsynaptic striatal metabotropic $\mathrm{GABA}_{\mathrm{B}}$ receptors that have a long decay time, resulting in reduced release of glutamate in the striatum and consequently reduced striatal excitation shortly after a tic (Calabresi et al., 1991; Nisenbaum et al., 1993; Shao and Burkhalter, 1999; Pérez-Garci et al., 2006; Logie et al., 2013). Thus, both proposed external and 
A

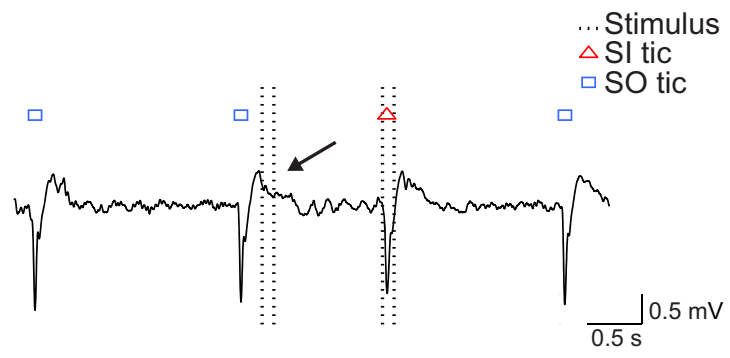

B i)

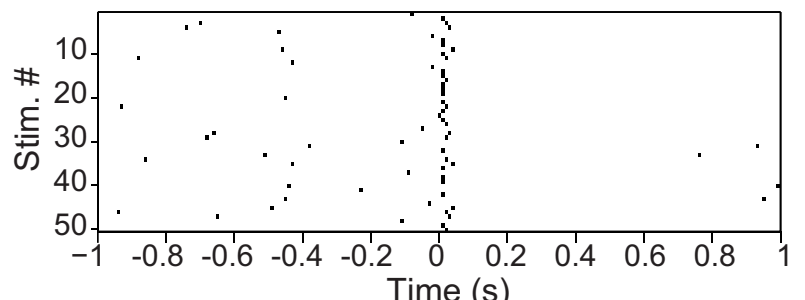

ii)

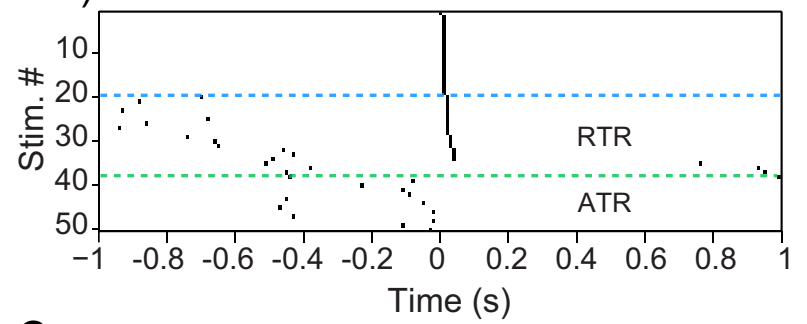

C
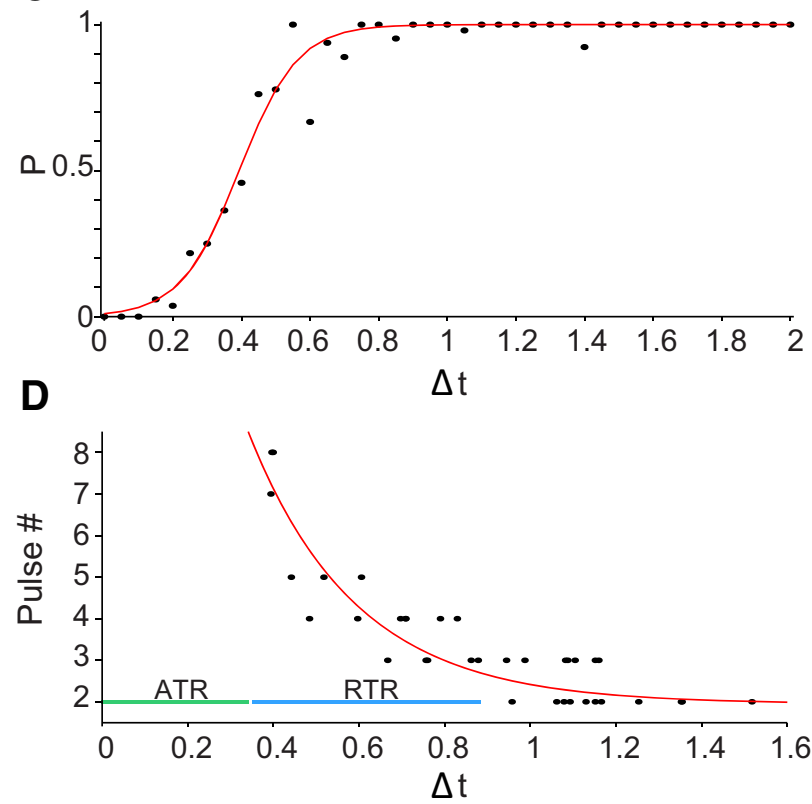

Figure 7. Absolute and partial tic refractory periods. A, A short trace of LFP illustrating a stimulation burst that did not induce an $\mathrm{SI}$ tic (arrow) as a result of a previous $\mathrm{SO}$ tic. $\boldsymbol{B}$, Peristimulus tic raster in trial order $(i)$ and the same raster sorted according to the latency between the stimulation onset and the induced tic (ii). C, The mean probability of SI ticformation as a function of latency from the previous tic (bin size, $0.05 \mathrm{~s}$ ). $\Delta t$, Time difference from stimulation onset to the last tic preceding it (s); red line, sigmoid curve fitting. $\boldsymbol{D}$, The number of stimulation pulses required to elicit an $\mathrm{SI}$ tic as a function of latency from the previous tic in one session. Red line, Exponential curve fitting.

internal neuronal mechanisms may play a role and intermingle to produce the observed striatal refractory periods.

The tic-generating network is based on the interplay among incoming inputs that are limited by refractory periods, much
A

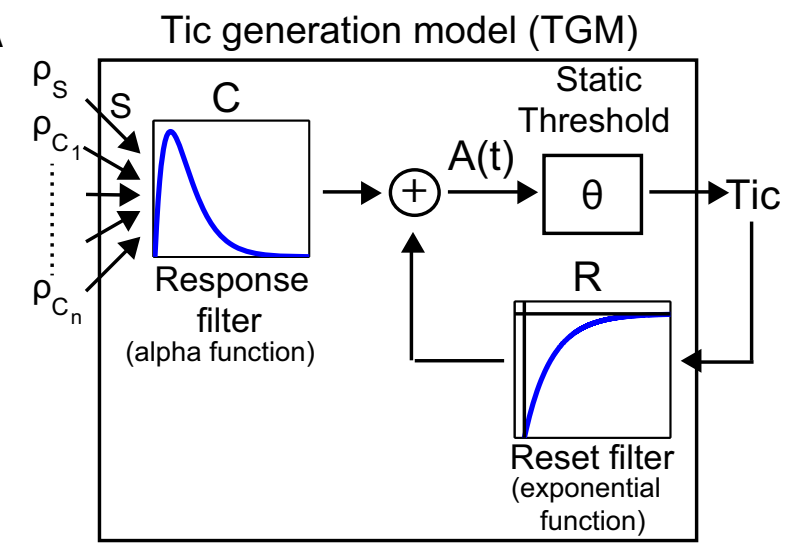

B

... Stimulus

$\triangle$ SI tic

$\square$ SO tic

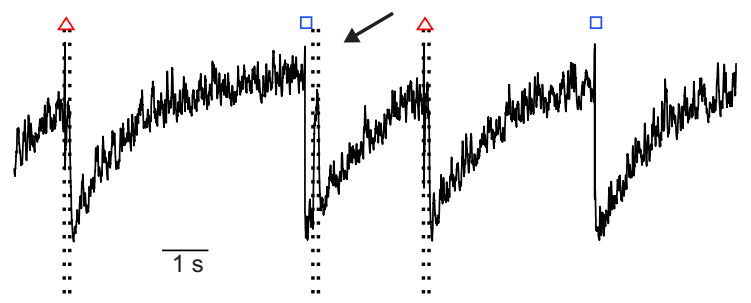

C

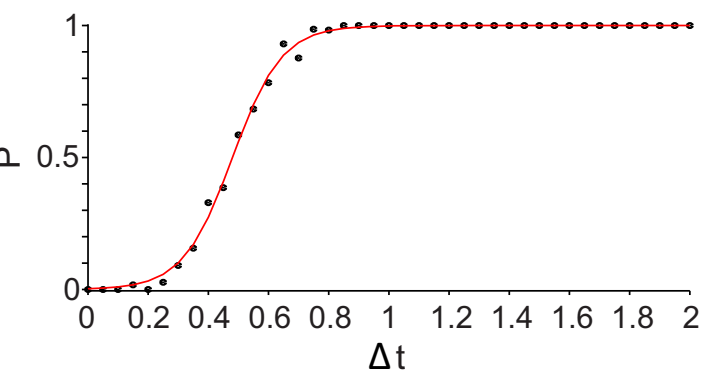

D

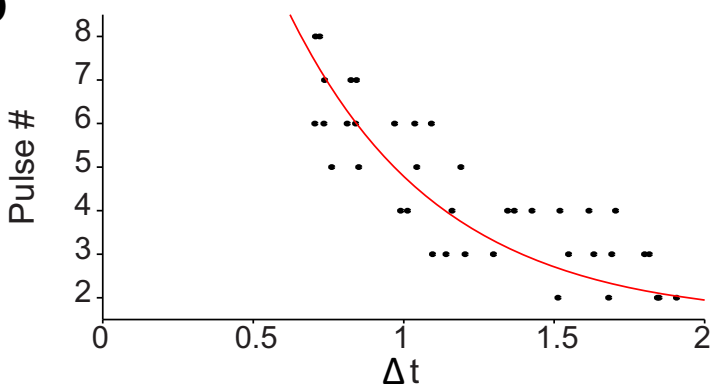

Figure 8. Tic generation model. $\boldsymbol{A}$, Schematic illustration of the TGM. $\boldsymbol{B}$, Example of TGM generated tics during a stimulation period. The arrow points to a stimulation burst that did not induce an $\mathrm{SI}$ tic as a result of a preceding $\mathrm{SO}$ tic. C, The mean probability of a stimulation leading to a tic as a function of latency from the preceding tic (bin size, $0.05 \mathrm{~s}$ ). Red line, Sigmoid curve fitting. $\boldsymbol{D}$, The number of stimulation pulses required to elicit an $\mathrm{SI}$ tic as a function of latency from the previous tic (random tics are presented). Red line, Exponential curve fitting. The simulation parameters used throughout the figure: $A_{R}=0.7, A_{C}=0.01 ; \tau_{R}=1400 \mathrm{~ms}, \tau_{c}=10$ $\mathrm{ms}, \mathrm{S}=3$, stimulation burst: 20 stimuli, $167 \mathrm{~Hz}$.

like single neurons; however, this network functions over significantly longer timescales (seconds compared with milliseconds). This observation was further supported by the results of the simple computational model (TGM) presented above based on a simplified $\mathrm{SRM}_{0}$ for neuronal activity (Gerstner, 1995). This phenomenological model quantifies the cortical activity effect on tic formation and illustrates the dependence 


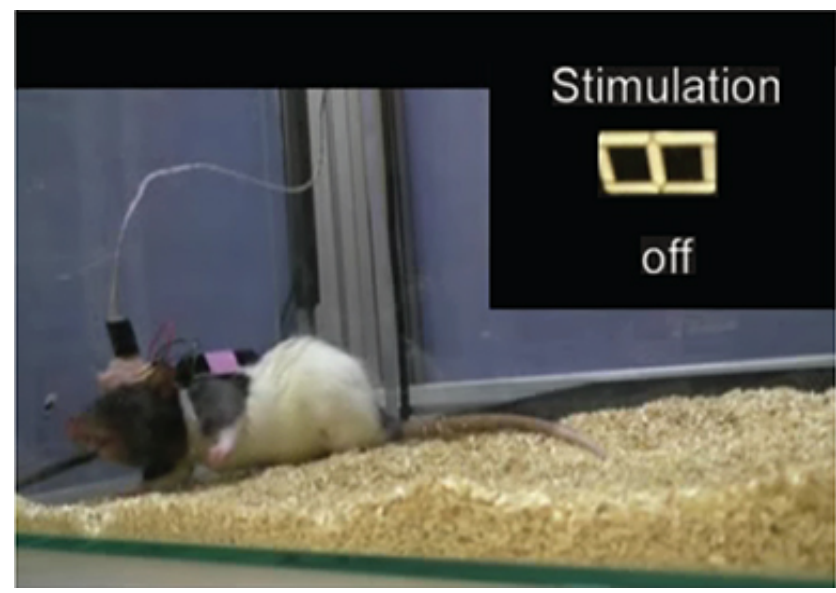

Movie 1. Tic expression after bicuculline injection. The injection of bicuculline leads to a tic expression period (TP), which consists of spontaneous (SO) tics and M1 forelimb stimulationinduced (SI) tics. The red light (top right) indicates the stimulation times.

\section{M1 stimulation - hindlimb}

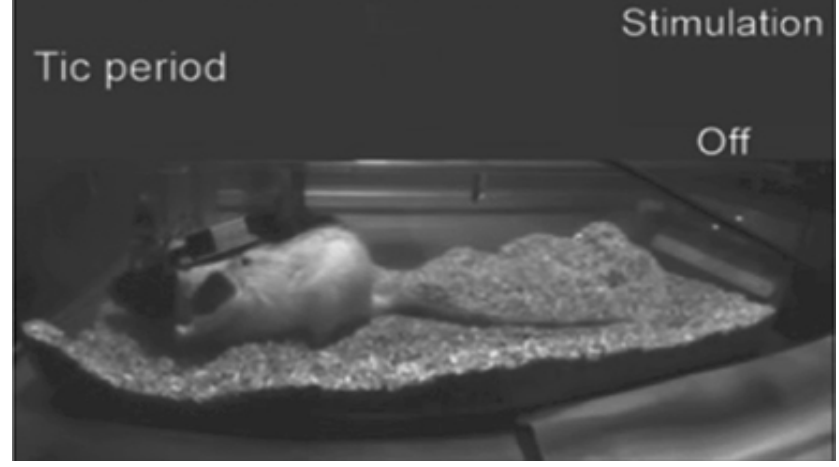

Movie 2. Differential effects of stimulation in the hindlimb somatotopic area of M1. A, Hindlimb cortical stimulation during the normal period (NP) leads to a contraction of the contralateral hindlimb. B, Hindlimb cortical stimulation during the tic period (TP) induces tics in the contralateral forelimb following bicuculline injection to the forelimb somatotopic region within the striatum. $C$, Hindlimb cortical stimulation during the post tic period again leads to a contraction of the contralateral hindlimb, similar to the period preceding the injection $(\boldsymbol{A})$.

of the tic time solely on the time of the previous tic and not on the whole tic history. Given this simplified assumption, the timing of the tics becomes highly predictable by the model, and the statistical properties of artificially generated tics resemble those of the experimentally induced ones. In the animal model, a reduction in the magnitude of the cortical input, for instance after a motor cortex lesion, abolished tic appearance but not the LFP spikes (Muramatsu et al., 1990), suggesting that tic timing may also be affected by other inputs to the striatum. This input may arise from other cortical and noncortical sources, such as glutamatergic inputs from the thalamus and dopaminergic inputs from the substantia nigra pars compacta (Parent and Hazrati, 1995; Haber et al., 2000; McFarland and Haber, 2000; Almeida et al., 2015). These additional sources may be added to the TGM by incorporating additional input with Poisson (or other) spike distributions feeding through separate response filters.

Tic generation has been linked to cortical activity in human TS patients using both fMRI (Neuner et al., 2014) and EEG (Bour et al., 2015). Enhanced motor cortex activity was observed up to $2 \mathrm{~s}$ before tic onset (Neuner et al., 2014). This result is consistent with our findings that, during TS, tic generation is preceded by increased cortical inputs to the striatum. Notably, despite the fact that tics are preceded by cortical activity, daily movements do not induce tics in TS patients. In our animal model as well, rat locomotion and overall movements did not alter tic frequency. This may suggest that strong activation of the cortex, such as after electrical stimulation or massive accumulation of inputs to the striatum, are required to induce a tic, whereas normal activities, such as walking, do not suffice in themselves. During the TP, excitation in cortical somatotopic regions that are not mapped to the tic expression region nevertheless induced a tic, with reduced or even without muscle contraction at the somatotopically excited region. This result may be accounted for by a study on TS patients that found that corticospinal excitability was reduced (Orth et al., 2008). Thus, in our model, corticospinal neurons related to somatotopic regions unassociated to the tic may be less excitable during the TP.

Our findings provide new insights into basic issues concerning BG normal function, such as the way that information is transferred through the CBG loop and the degree of functional overlap between different cortical projecting regions. Our results suggest that the corticostriatal information flow is not completely parallel but rather has convergent and integrative properties. This notion is supported by recent studies on healthy humans showing the existence of overlapping projections (Draganski et al., 2008; Oguri et al., 2013). In addition, our results shed light on the BG contribution to movement by suggesting that the BG play a critical role in action execution and by supporting the notion that a subset of striatal neurons encode a specific action (Mink, 1996). According to this reasoning, corticostriatal projections excite a subset of striatal neurons that leads to a release of the selected action. The selection process of the action within the striatum involves both feedback and feedforward inhibition that inhibits all other potential actions. In TS, the normal processing in the BG is disrupted by striatal disinhibition that decreases the excitation threshold of the neurons located in the disinhibited region, leading to an abnormal release of the action (tic; Mink, 2001; Albin and Mink, 2006). This notion is supported by postmortem findings of reduced striatal modulation by multiple interneurons and the GP (Kalanithi et al., 2005; Kataoka et al., 2010) that is mimicked experimentally by focal $\mathrm{GABA}_{\mathrm{A}}$ antagonists. Our results augment this basic concept of action release by demonstrating a clear functional dichotomy within the corticostriatal circuit. Our data suggest that, during normal function, the striatal network selects the specifics of the action ("where"), whereas the corticostriatal activity determines the time ("when") of the action. Thus, the temporal and spatial properties of the action release have disparate encoding.

The two key questions related to tic expression in TS and other tic disorders are the "when" and "where" questions. The striatal disinhibition animal model made it possible to establish the pivotal role of the somatotopic location of the deficit within the striatum in determining the body region expressing the tic and thus addresses the "where" question (Bronfeld et al., 2013). The current study complemented this picture by revealing a possible answer to the "when" question. According to our findings, tics originate in the striatum, and their timing depends on the interplay between incoming excitatory inputs and the internal striatal state. Thus, whereas the striatum determines the spatial properties of actions, the cortex plays a 
major role in determining the temporal properties. This may hint that control over corticostriatal transmission may reduce tics and serve as a possible treatment for TS patients. Finally, this study underscores a key issue related to basic BG functioning; namely that, during normal function, the cortex determines the timing of the action processes via the CBG pathway but not the properties of this action, whereas the striatum determines the specific action. Thus, the combination of striatal disinhibition with fine temporal control over cortical excitation leads to novel insights into the release of actions by the CBG pathway in health and disease.

\section{Notes}

Supplemental material for this article is available at http://neurint.ls.biu. ac.il/ArticlesMultimedia.html. This material has not been peer reviewed.

\section{References}

Albin RL, Mink JW (2006) Recent advances in Tourette syndrome research. Trends Neurosci 29:175-182. CrossRef Medline

Almeida L, Martinez-Ramirez D, Rossi PJ, Peng Z, Gunduz A, Okun MS (2015) Scheduled and closed loop responsive approaches. J Clin Neurol 11:122-131. CrossRef Medline

American Psychiatric Association (2013) DSM-5. Arlington, VA: American Psychiatric Association.

Benhamou L, Bronfeld M, Bar-Gad I, Cohen D (2012) Globus pallidus external segment neuron classification in freely moving rats: a comparison to primates. PLoS One 7:e45421. CrossRef Medline

Bennett BD, Bolam JP (1994) Synaptic input and output of parvalbuminimmunoreactive neurons in the neostriatum of the rat. Neuroscience 62:707-719. CrossRef Medline

Bloch MH, Leckman JF, Zhu H, Peterson BS (2005) Caudate volumes in childhood predict symptom severity in adults with Tourette syndrome. Neurology 65:1253-1258. CrossRef Medline

Bour LJ, Ackermans L, Foncke EM, Cath D, van der Linden C, Visser Vandewalle V, Tijssen MA (2015) Tic related local field potentials in the thalamus and the effect of deep brain stimulation in Tourette syndrome: report of three cases. Clin Neurophysiol 126:1578-1588. Medline

Bronfeld M, Belelovsky K, Bar-Gad I (2011) Spatial and temporal properties of tic-related neuronal activity in the cortico-basal ganglia loop. J Neurosci 31:8713-8721. CrossRef Medline

Bronfeld M, Yael D, Belelovsky K, Bar-Gad I (2013) Motor tics evoked by striatal disinhibition in the rat. Front Syst Neurosci 7:50. CrossRef Medline

Calabresi P, Mercuri NB, De Murtas M, Bernardi G (1991) Involvement of GABA systems in feedback regulation of glutamate-and GABA-mediated synaptic potentials in rat neostriatum. J Physiol 440:581-599. CrossRef Medline

Crossman AR, Mitchell IJ, Sambrook MA, Jackson A (1988) Chorea and myoclonus in the monkey induced by gamma-aminobutyric acid antagonism in the lentiform complex. The site of drug action and a hypothesis for the neural mechanisms of chorea. Brain 111:1211-1233. CrossRef Medline

Draganski B, Kherif F, Klöppel S, Cook PA, Alexander DC, Parker GJ, Deichmann R, Ashburner J, Frackowiak RS (2008) Evidence for segregated and integrative connectivity patterns in the human basal ganglia. J Neurosci 28:7143-7152. CrossRef Medline

Erez Y, Tischler H, Moran A, Bar-Gad I (2010) Generalized framework for stimulus artifact removal. J Neurosci Methods 191:45-59. CrossRef Medline

Gerstner W (1995) Time structure of the activity in neural network models. Phys Rev E Stat Phys Plasmas Fluids Relat Interdiscip Topics 51:738-758. Medline

Haber SN, Fudge JL, McFarland NR (2000) Striatonigrostriatal pathways in primates form an ascending spiral from the shell to the dorsolateral striatum. J Neurosci 20:2369-2382. Medline

Inghilleri M, Berardelli A, Cruccu G, Manfredi M (1993) Silent period evoked by transcranial stimulation of the human cortex and cervicomedullary junction. J Physiol 466:521-534. Medline

Kalanithi PS, Zheng W, Kataoka Y, DiFiglia M, Grantz H, Saper CB, Schwartz ML, Leckman JF, Vaccarino FM (2005) Altered parvalbumin-positive neuron distribution in basal ganglia of individuals with Tourette syndrome. Proc Natl Acad Sci U S A 102:1330713312. CrossRef Medline

Kataoka Y, Kalanithi PS, Grantz H, Schwartz ML, Saper C, Leckman JF, Vaccarino FM (2010) Decreased number of parvalbumin and cholinergic interneurons in the striatum of individuals with Tourette syndrome. J Comp Neurol 518:277-291. CrossRef Medline

Koós T, Tepper JM (1999) Inhibitory control of neostriatal projection neurons by GABAergic interneurons. Nat Neurosci 2:467-472. CrossRef Medline

Krnjević K, Randić M, Straughan DW (1966) Nature of a cortical inhibitory process. J Physiol 184:49-77. Medline

Kujirai T, Caramia MD, Rothwell JC, Day BL, Thompson PD, Ferbert A, Wroe S, Asselman P, Marsden CD (1993) Corticocortical inhibition in human motor cortex. J Physiol 471:501-519. Medline

Lerner A, Bagic A, Simmons JM, Mari Z, Bonne O, Xu B, Kazuba D, Herscovitch P, Carson RE, Murphy DL, Drevets WC, Hallett M (2012) Widespread abnormality of the $\gamma$-aminobutyric acid-ergic system in Tourette syndrome. Brain 135:1926-1936. CrossRef Medline

Logie C, Bagetta V, Bracci E (2013) Presynaptic control of corticostriatal synapses by endogenous GABA. J Neurosci 33:15425-15431. CrossRef Medline

Mallet N, Micklem BR, Henny P, Brown MT, Williams C, Bolam JP, Nakamura KC, Magill PJ (2012) Dichotomous organization of the external globus pallidus. Neuron 74:1075-1086. CrossRef Medline

Marsden CD, Meldrum BS, Pycock C, Tarsy D (1975) Focal myoclonus produced by injection of picrotoxin into the caudate nucleus of the rat. J Physiol 246:96P. Medline

McCairn KW, Bronfeld M, Belelovsky K, Bar-Gad I (2009) The neurophysiological correlates of motor tics following focal striatal disinhibition. Brain 132:2125-2138. CrossRef Medline

McCairn KW, Iriki A, Isoda M (2013) Global dysrhythmia of cerebro-basal ganglia-cerebellar networks underlies motor tics following striatal disinhibition. J Neurosci 33:697-708. CrossRef Medline

McFarland NR, Haber SN (2000) Convergent inputs from thalamic motor nuclei and frontal cortical areas to the dorsal striatum in the primate. J Neurosci 20:3798-3813. Medline

Mink JW (1996) The basal ganglia: Focused selection and inhibition of competing motor programs. Prog Neurobiol 50:381-425. CrossRef Medline

Mink JW (2001) Basal ganglia dysfunction in Tourette's syndrome: a new hypothesis. Pediatr Neurol 25:190-198. CrossRef Medline

Muramatsu S, Yoshida M, Nakamura S (1990) Electrophysiological study of dyskinesia produced by microinjection of picrotoxin into the striatum of the rat. Neurosci Res 7:369-380. CrossRef Medline

Neuner I, Werner CJ, Arrubla J, Stöcker T, Ehlen C, Wegener HP, Schneider F, Shah NJ (2014) Imaging the where and when of tic generation and resting state networks in adult Tourette patients. Front Hum Neurosci 8:362. CrossRef Medline

Nisenbaum ES, Berger TW, Grace AA (1993) Depression of glutamatergic and GABAergic synaptic responses in striatal spiny neurons by stimulation of presynaptic GABAB receptors. Synapse 14:221-242. CrossRef Medline

Oguri T, Sawamoto N, Tabu H, Urayama S, Matsuhashi M, Matsukawa N, Ojika K, Fukuyama H (2013) Overlapping connections within the motor cortico-basal ganglia circuit: fMRI-tractography analysis. Neuroimage 78:353-362. CrossRef Medline

Orth M, Münchau A, Rothwell JC (2008) Corticospinal system excitability at rest is associated with tic severity in Tourette syndrome. Biol Psychiatry 64:248-251. CrossRef Medline

Parent A, Hazrati LN (1995) Functional anatomy of the basal ganglia. I. The cortico-basal ganglia-thalamo-cortical loop. Brain Res Rev 20:91-127. CrossRef Medline

Paxinos G, Watson C (2007) The rat brain in stereotaxic coordinates. Academic: San Diego.

Pérez-Garci E, Gassmann M, Bettler B, Larkum ME (2006) The GABAB1b isoform mediates long-lasting inhibition of dendritic $\mathrm{Ca}^{2+}$ spikes in layer 5 somatosensory pyramidal neurons. Neuron 50:603-616. CrossRef Medline

Peterson BS, Thomas P, Kane MJ, Scahill L, Zhang H, Bronen R, King RA, Leckman JF, Staib L (2003) Basal ganglia volumes in patients with 
Gilles de la Tourette syndrome. Arch Gen Psychiatry 60:415-424. CrossRef Medline

Pogorelov V, Xu M, Smith HR, Buchanan GF, Pittenger C (2015) Corticostriatal interactions in the generation of tic-like behaviors after local striatal disinhibition. Exp Neurol 265:122-128. CrossRef Medline

Shao Z, Burkhalter A (1999) Role of GABAB receptor-mediated inhibition in reciprocal interareal pathways of rat visual cortex. J Neurophysiol 81: 1014-1024. Medline

Singer HS, Minzer K (2003) Neurobiology of Tourette's syndrome: concepts of neuroanatomic localization and neurochemical abnormalities. Brain Dev 25:S70-S84. CrossRef Medline

Tarsy D, Pycock CJ, Meldrum BS, Marsden CD (1978) Focal contralateral myoclonus produced by inhibition of GABA action in the caudate nucleus of rats. Brain 101:143-162. CrossRef Medline

Tischler H, Moran A, Belelovsky K, Bronfeld M, Korngreen A, Bar-Gad I (2012) Changes in basal ganglia processing of cortical input following magnetic stimulation in Parkinsonism. Neurobiol Dis 48:464-473. CrossRef Medline

Wang Z, Maia TV, Marsh R, Colibazzi T, Gerber A, Peterson BS (2011) The neural circuits that generate tics in Tourette's syndrome. Am J Psychiatry 168:1326-1337. CrossRef Medline

Werhahn KJ, Kunesch E, Noachtar S, Benecke R, Classen J (1999) Differential effects on motorcortical inhibition induced by blockade of GABA uptake in humans. J Physiol 517:591-597. CrossRef Medline

Yael D, Zeef DH, Sand D, Moran A, Katz DB, Cohen D, Temel Y, Bar-Gad I (2013) Haloperidol-induced changes in neuronal activity in the striatum of the freely moving rat. Front Syst Neurosci 7:110. CrossRef Medline

Yoshida M, Nagatsuka Y, Muramatsu S, Niijima K (1991) Differential roles of the caudate nucleus and putamen in motor behavior of the cat as investigated by local injection of GABA antagonists. Neurosci Res 10: 34-51. CrossRef Medline 